\title{
Lifetime cognition and late midlife blood metabolites: findings from a British birth cohort
}

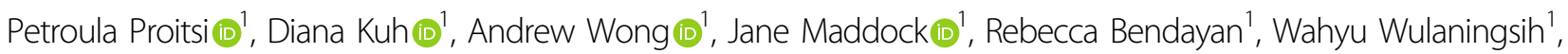 \\ Rebecca Hardy ${ }^{1}$ and Marcus Richards ${ }^{1}$
}

\begin{abstract}
Maintenance of healthy cognitive ageing is vital for independence and wellbeing in the older general population. We investigated the association between blood metabolites and cognitive function and decline. Participants from the MRC National Survey of Health and Development (NSHD, the British 1946 birth cohort) were studied; 233 nuclear magnetic resonance circulating metabolite measures were quantified in 909 men and women at ages 60-64. Shortterm and delayed verbal memory and processing speed were concurrently assessed and these tests were repeated at age 69. Linear regression analyses tested associations between metabolites and cognitive function at ages 60-64, and changes in these measures by age 69, adjusting for childhood cognition, education, socio-economic status and lifestyle factors. In cross-sectional analyses, metabolite levels, particularly fatty acid composition and different lipid subclasses, were associated with short-term verbal memory (4 measures in females and 11 measures in the whole sample), delayed verbal memory ( 2 measures in females) and processing speed ( 8 measures in males and 2 measures in the whole sample) $(p<0.002)$. One metabolite was associated with change in cognition in females. Most of the observed associations were attenuated after adjustment for childhood cognition and education. A life course perspective can improve the understanding of how peripheral metabolic processes underlie cognitive ageing.
\end{abstract}

\section{Introduction}

Global increases in life expectancy have been accompanied by changes in labour market and social structures that place growing importance on the maintenance of healthy cognitive ageing for independence and wellbeing in the older general population ${ }^{1}$. It is therefore important to identify ways to maintain healthy cognitive ageing and to prevent or delay functionally significant cognitive decline, especially in the absence of effective diseasemodifying treatments for dementia.

Blood metabolites closely represent the physiological status of an organism, reflecting what has been encoded by the genome and modified by systemic and environmental exposures ${ }^{2}$. Markers of lipid metabolism, such as

Correspondence: Petroula Proitsi (p.proitsi@ucl.ac.uk)

${ }^{1} \mathrm{MRC}$ Unit for Lifelong Health and Ageing at UCL, London, UK essential fatty acids (FAs) and in particular omega-3 polyunsaturated FAs (n3-PUFAs), play a vital structural and functional role in the central nervous system, and are associated with cognitive performance and brain function during general ageing ${ }^{3,4}$. However, most studies linking lipids to cognitive ageing are limited by inability to control for potential reverse causality, since associations between lipids and cognition reflect lifetime interplay.

The MRC National Survey of Health and Development (NSHD, the British 1946 birth cohort) offers unique opportunities using an age-homogenous sample. Study members are now at an age where pathophysiological changes are likely to be accumulating, but frank dementia is still rare. A wide range of blood metabolites was assayed in late midlife (between ages 60 and 64) using nuclear magnetic resonance (NMR), and memory and processing speed were concurrently assessed. These cognitive tests 
were repeated at age 69 . In addition, the NSHD has a wide range of potential confounders and mediators, including childhood cognitive ability ${ }^{5}$, education, lifetime socioeconomic position, and health and health-related behaviours. We investigated blood metabolite levels in relation to cognitive function and decline.

\section{Materials and methods Participants}

The Medical Research Council (MRC) National Survey of Health and Development (NSHD) is based on a nationally representative cohort of 5362 singleton births within marriage occurring during 1 week in March 1946 in England, Scotland and Wales. The cohort has been followed 24 times, most recently when participants were 68-69 years old $^{6}$. Extensive information on sociodemographics, health and cognitive function has been obtained in childhood, adolescence and regularly thereafter ${ }^{6}$.

For the 60-64 years wave, 2229 of 2856 eligible participants (78.0\%) underwent assessment. Contact was not attempted with those who were known to have died $(n=$ $778)$, were living abroad $(n=570)$, had previously withdrawn from the study $(n=594)$ or were permanently untraced $(n=564)^{7}$. Of those assessed, 98\% were willing to have a blood sample taken, and at least one blood sample was successfully obtained from $96 \%$.

The participating sample remains broadly representative of native-born British men and women of the same age $^{7}$. The current study protocol received ethical approval from the Greater Manchester Local Research Ethics Committee for the four English sites and from the Scotland A Research Ethics Committee. Written informed consent was obtained at each data collection.

\section{Metabolomics}

Serum metabolomics analyses were performed on blood samples collected at ages 60-64. All blood samples were collected after an overnight fast and were not subjected to any free-thaw cycles prior to metabolomics. Serum metabolites were assayed using a high-throughput NMR metabolomics platform able to quantify up to 233 metabolite measures and ratios representing a broad molecular signature of systemic metabolism. Multiple metabolic pathways were covered, including lipoprotein lipids and lipid sub-classes, FAs and FA compositions, as well as amino acids and glycolysis precursors (Supplementary Table S1). Details are described elsewhere ${ }^{8-10}$. Following strict quality control (QC) serum metabolite data were available for 909 participants.

\section{Cognitive function}

Cognitive function was assessed by short-term and delayed verbal memory, and speed of processing at age
60-64, and by change in these measures (except delayed verbal memory) by age 69 . Short-term verbal memory was assessed by a three-trial 15 -item word list learning task (maximum score $=45$ ) devised by the $\mathrm{NSHD}^{11}$. After a processing speed task (see below), an uncued delayed free recall trial was administered (delayed verbal memory; 60-64 years only). Speed of processing was assessed as the number of letters $\mathrm{P}$ and $\mathrm{W}$, randomly embedded within a page of other letters, crossed out as quickly and accurately as possible within $1 \mathrm{~min}$ (maximum 600$)^{11}$.

\section{Covariables}

The following variables were treated as potential confounders or mediators: sex, age at blood collection and blood collection centre, cognitive ability at 15 years, educational attainment and childhood and midlife SEP, BMI at 60-64 years ${ }^{12}$, lipid medication, lifetime smoking and alcohol consumption by $60-64$ years, and exercise and nutrient intakes at 60-64 years ${ }^{13-16}$.

Cognitive ability at 15 years was represented as the sum of four tests of verbal and nonverbal ability ${ }^{17}$. Educational attainment by 26 years was grouped in three categories: no educational qualifications, ordinary ('O' level) secondary qualifications and advanced ('A' level) secondary or higher qualifications. Lifetime SEP was based on father's occupation when study members were aged 11 (or if this was unknown at ages 4 or 15) and current or last own occupation at age 53; both were coded in six categories according to the UK Registrar General's classification. Weight and standing height were measured at 60-64 years according to standard protocols, and BMI was calculated. Lipid medication was recorded by a research nurse as any lipid lowering drugs taken in the last $24 \mathrm{~h}$ before the blood sample was taken.

Lifetime smoking was represented by pack years per person from 20 to 64 years. Physical activity was defined as participating in any sports, exercises or vigorous leisure activities in the month preceding the age 60-64 interview (none, 1-4 times or more than 4 times). Participants recorded all alcoholic drinks consumed using 3-5 day diet diaries at $36,43,53$ and $60-64$ years $^{18}$. An overall measure of adult alcohol consumption was calculated as the average of daily intakes (in grams) at all ages (when data were available for at least three of four waves); this was recoded as no consumption, light to moderate and heavy consumption across midlife.

Diet at 60-64 years was assessed using 5-day estimated diet diaries ${ }^{19}$. Mean daily consumption of the following nutrients were calculated when data was available for at least three days: total carbohydrates, total fat and total saturated FAs, total mono-unsaturated FAs, n3-PUFAs and n6-PUFAs, including any supplements taken; and nutrient densities per $1000 \mathrm{kcal}$ were generated (grams/ total energy $(\mathrm{kcal}) \times 1000)$. 


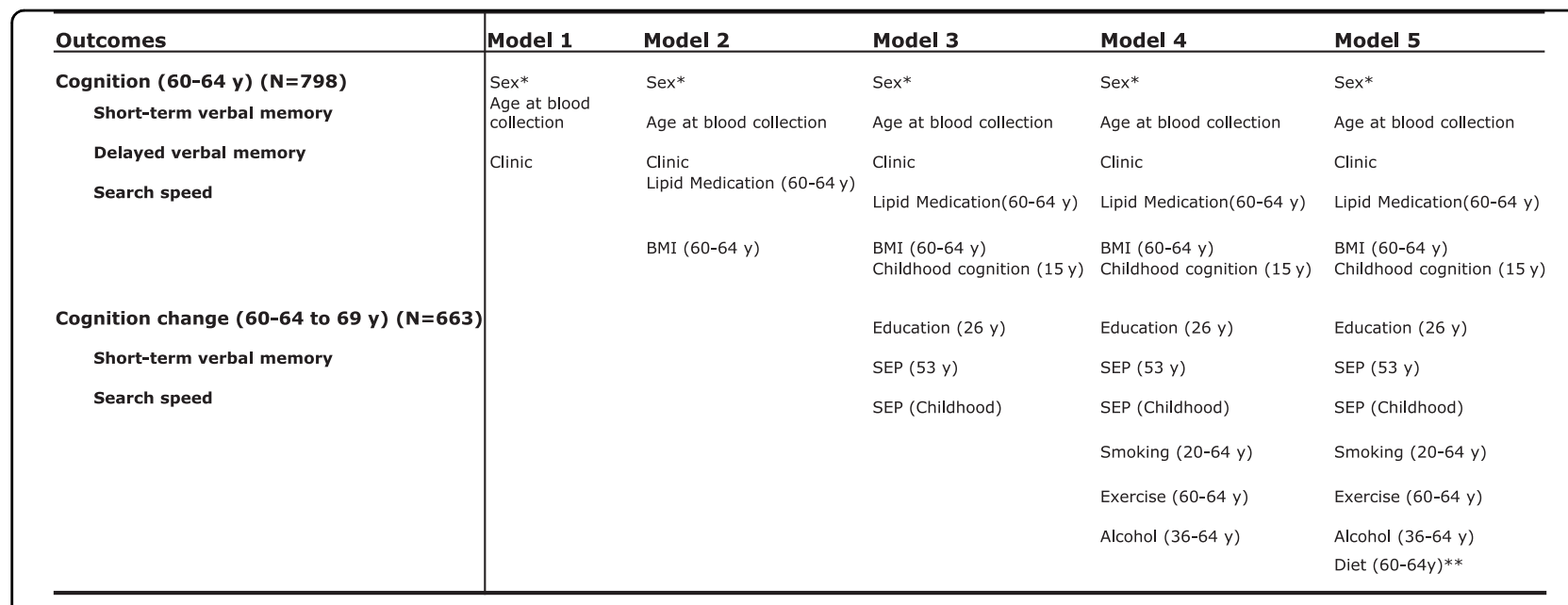

Fig. 1 Stages of covariate adjustment. *Sex adjustment took place for whole-sample analyses. **Model 5 was run 6 times, using a different nutrient intake variable in each model (Table 1)

\section{Statistical analyses}

The outcomes of the study were the cognitive variables and the predictors were the metabolic measures. All cognitive variables were approximately normally distributed. Metabolite measures that showed any deviations from normality were transformed using the natural logarithm, or the natural logarithm plus 0.1 if they included zero values (Supplementary Table S1) n3-PUFAs and n6-PUFAs nutrient densities were skewed, so natural log transformed. Upon transformation all variables were approximately normally distributed. All outcomes and predictors were standardised for direct comparisons.

\section{Main analyses}

Linear regression was used to test associations between metabolites and memory and search speed. There were five stages of adjustments (Fig. 1): Model 1 adjusted for sex, age at blood collection and blood collection centre; Model 2 additionally adjusted for BMI and lipid medication at 60-64 years; Model 3 further adjusted for cognitive ability, educational attainment and lifetime SEP; Model 4 further adjusted for lifetime smoking, alcohol consumption and exercise at 60-64 years; Model 5 additionally adjusted for nutrient intake at 60-64 years; Model 5 was rerun 6 times including a different diet variable at each model. To maintain statistical power and minimise bias from missing data, we imputed missing values for the covariables in the sample of 798 participants with complete metabolite and cognition data at 60-64 years using multiple imputation chained equations (mice) implemented in $R^{20}$. Sex interactions were tested in Model 1 and all models were sex-stratified when there was evidence of significant sex interaction $(p<0.1)$.
In the sample with a cognitive measure at age 69, we repeated this series of models for change (except delayed verbal memory) between age 60-64 and 69, by taking the difference between age 60-64 and 69 and adjusting for baseline (age 60-64).

To correct for multiple testing and correlation between metabolic measures, we set a metabolome-wide statistical significance threshold to $p<0.002$ (Supplementary Figure $\mathrm{S} 1$ ); the $p<0.05$ significance level was divided by the number of principal components ${ }^{25}$ that explained over $95 \%$ of variation in the metabolomics data. All analyses were conducted in R.3.1.1

\section{Additional analyses}

In preliminary analyses we investigated associations between all covariables and the metabolites in the whole sample (adjusted for sex, age at blood collection and blood collection clinic) and in females and males separately (adjusted for age at blood collection and blood collection clinic) (Supplementary Table S2).

We performed sensitivity analyses by adjusting for one covariable at a time in addition to the Model 1 covariables (Supplementary Table S3 (a-c)). We also compared those with complete data through Model 3, to those with complete data on all covariates.

\section{Results}

\section{Study sample characteristics}

Of the 909 study participants with metabolite data, 798 had complete data at ages 60-64 for metabolite and cognitive measures. Of these, 663 participants had repeat cognitive measures at age 69. Characteristics of the samples are shown in Table 1. 
Table 1 Characteristics of participants with complete data for all metabolites and cognitive measures at 60-64 years $(N=798)$ and both at $60-64$ and 69 years $(N=663)$

\begin{tabular}{|c|c|c|c|c|c|c|}
\hline & \multicolumn{3}{|c|}{$\begin{array}{l}\text { Complete metabolite and cognition } \\
\text { data for } 60-64 \text { y }\left(N_{\max }=798\right)\end{array}$} & \multicolumn{3}{|c|}{$\begin{array}{l}\text { Complete metabolite and cognition } \\
\text { data for } 60-64 \text { and } 69 \text { y }\left(N_{\max }=663^{\mathrm{a}}\right)\end{array}$} \\
\hline & All & Women & Men & All & Women & Men \\
\hline SEP (Father's), $n$ (\%) & $N=768$ & $N=400$ & $N=427$ & $N=639$ & $N=312$ & $N=327$ \\
\hline Professional & & $26(6.9)$ & $27(6.9)$ & & $23(7.4)$ & $25(7.6)$ \\
\hline Intermediate & & $78(20.7)$ & $96(24.6)$ & & $64(20.5)$ & $84(25.7)$ \\
\hline Nonmanual skilled & & $62(16.5)$ & $58(15.5)$ & & $52(16.6)$ & $50(15.3)$ \\
\hline Manual skilled & & $120(31.8)$ & $121(31.4)$ & & $102(32.7)$ & $102(31.2)$ \\
\hline Partly skilled & & $72(19.1)$ & $65(16.6)$ & & $59(18.9)$ & $47(14.4)$ \\
\hline Unskilled & & $19(5.0)$ & $24(6.1)$ & & $12(3.9)$ & $19(5.8)$ \\
\hline \multirow[t]{2}{*}{ Childhood cognition (15 y) (z-score), mean(SD) } & $N=705$ & $N=349$ & $N=356$ & $N=585$ & $N=288$ & $N=297$ \\
\hline & & $-0.06(0.96)$ & $0.06(1.03)$ & & $0.00(0.93)$ & $0.12(1.02)$ \\
\hline Education (26y), $n$ (\%) & $N=760$ & $N=377$ & $N=383$ & $N=633$ & $N=314$ & $N=319$ \\
\hline No qualification & & $112(29.7)$ & $118(30.8)$ & & $91(28.9)$ & $88(27.5)$ \\
\hline Up to GCSE & & $126(33.4)$ & $74(19.3)$ & & $103(32.8)$ & $62(19.4)$ \\
\hline A-level or higher & & $139(36.9)$ & $191(48.9)$ & & $120(38.2)$ & $169(53.0)$ \\
\hline $\operatorname{SEP}(15-53 \mathrm{y}), n(\%)$ & $N=796$ & $N=390$ & $N=406$ & $N=662$ & $N=324$ & $N=338$ \\
\hline Professional & & $6(1.54)$ & $58(14.3)$ & & $5(1.54)$ & $50(14.8)$ \\
\hline Intermediate & & $164(42.1)$ & $180(44.3)$ & & $145(44.8)$ & $155(45.9)$ \\
\hline Nonmanual skilled & & $128(32.8)$ & $39(9.6)$ & & $105(32.4)$ & $35(10.4)$ \\
\hline Manual skilled & & $34(8.7)$ & $97(23.9)$ & & $25(7.7)$ & $72(21.3)$ \\
\hline Partly skilled & & $46(11.8)$ & $23(5.7)$ & & $34(10.5)$ & $19(5.5)$ \\
\hline Unskilled & & $12(3.08)$ & $9(2.2)$ & & $10(3.09)$ & $7(2.1)$ \\
\hline \multirow[t]{2}{*}{ BMI (64 y) (weight $(\mathrm{kg}) /$ height $\left.(\mathrm{m})^{2}\right)$, mean(SD) } & $N=797$ & $N=389$ & $N=498$ & $N=662$ & $N=323$ & $N=339$ \\
\hline & & $27.54(4.7)$ & $27.4(3.9)$ & & $27.64(4.8)$ & $27.2(3.8)$ \\
\hline Lipid Medication (64 y), $n$ (\%) & $N=798$ & $N=390$ & $N=408$ & $N=663$ & $N=324$ & $N=339$ \\
\hline Yes & & $68(17.4)$ & $105(25.7)$ & & $63(19.4)$ & $86(25.4)$ \\
\hline No & & $322(82.6)$ & $322(74.3)$ & & $261(89.6)$ & $253(74.6)$ \\
\hline Physical activity (64 y), $n$ (\%) & $N=769$ & $N=371$ & $N=398$ & $N=643$ & $N=312$ & $N=331$ \\
\hline None & & $215(58.0)$ & $247(62.1)$ & & $176(56.4)$ & $191(57.7)$ \\
\hline $1-4$ times a month & & $56(15.0)$ & $59(14.8)$ & & $50(16.0)$ & $55(16.6)$ \\
\hline $4+$ times a month & & $100(27.0)$ & $92(21.1)$ & & $86(27.6)$ & $85(25.7)$ \\
\hline Lifetime smoking (20-64 y), $n$ (\%) & $N=645$ & $N=310$ & $N=335$ & $N=534$ & $N=256$ & $N=278$ \\
\hline Pack years per person & & $10.83(15.3)$ & $13.25(17.5)$ & & $10.12(15.2)$ & $12.08(16.9)$ \\
\hline Lifetime alcohol consumption (36-64 y), $n$ (\%) & $N=668$ & $N=324$ & $N=344$ & $N=563$ & $N=270$ & $N=293$ \\
\hline No consumption & & $27(8.3)$ & $13(3.8)$ & & $22(8.2)$ & $9(3.1)$ \\
\hline Light-moderate consumption & & $277(85.5)$ & $252(73.3)$ & & $229(84.8)$ & $220(75.1)$ \\
\hline \multirow[t]{2}{*}{ Heavy consumption } & & $20(6.2)$ & $79(22.9)$ & & $19(7.0)$ & $64(21.8)$ \\
\hline & $N=680$ & $N=336$ & $N=344$ & $N=586$ & $N=283$ & $N=303$ \\
\hline
\end{tabular}


Table 1 continued

\begin{tabular}{|c|c|c|c|c|c|c|}
\hline & \multicolumn{3}{|c|}{$\begin{array}{l}\text { Complete metabolite and cognition } \\
\text { data for } 60-64 \text { y }\left(N_{\max }=798\right)\end{array}$} & \multicolumn{3}{|c|}{$\begin{array}{l}\text { Complete metabolite and cognition } \\
\text { data for } 60-64 \text { and } 69 \text { y }\left(N_{\max }=663^{\mathrm{a}}\right)\end{array}$} \\
\hline & All & Women & Men & All & Women & Men \\
\hline \multicolumn{7}{|c|}{$\begin{array}{l}\text { Diet }(64 \mathrm{y}) \text {, mean daily nutrient densities } / 1000 \mathrm{kcal} \text {, mean } \\
\text { (SD) }\end{array}$} \\
\hline Carbohydrates & & $118.53(18.1)$ & $114.99(18.7)$ & & $118.77(18.2)$ & $114.72(18.8)$ \\
\hline Fat & & $37.98(6.7)$ & $37.55(6.2)$ & & $38.01(6.9)$ & $37.57(6.2)$ \\
\hline Total saturated FAs (FA) & & $14.36(3.8)$ & $14.09(3.41)$ & & $14.31(3.7)$ & $14.08(3.5)$ \\
\hline Total mono-unsaturated FAs (MUFA) & & $12.53(2.7)$ & $12.74(2.6)$ & & $12.54(2.7)$ & $12.72(2.6)$ \\
\hline n3-polyunsaturated FAs (n3-PUFA) & & $1.09(0.4)$ & $1.04(0.4)$ & & $1.10(0.4)$ & $1.05(0.4)$ \\
\hline n6-polyunsaturated FAs (n6-PUFA) & & $5.74(1.8)$ & $5.41(1.8)$ & & $5.75(1.8)$ & $5.40(1.8)$ \\
\hline
\end{tabular}

${ }^{a}$ Out of the 135 participants who were not included in the analyses at age 69,45 were not approached as they had died ( $n=38$ ) or had been lost to follow-up ( $n=7$ ). The remainder 90 were approached but temporarily refused to participate $(n=42)$, did not respond $(n=19)$, withdrew $(n=4)$ or did not have full assessment completed $(n=25)$

\section{Cognition at $60-64$ years}

Short-term verbal memory

Of the eleven metabolite measures with significant sex modification $(p<0.1)$ in Model 1 , four were associated with short-term verbal memory in females after correction for multiple testing (Fig. 2, Model 1): omega-3 Fas (FAw3) and DHA and their ratios to total FAs (FAw3-FA and DHA-FA respectively); the strongest association was with DHA-FA (beta $=0.256,95 \%$ CI $0.16-0.36, p=$ $\left.4.94 \times 10^{-7}\right)$. Adjustment for BMI and lipid medication slightly reduced some of these associations (Model 2) but most were attenuated by childhood cognitive ability, education and SEP, particularly by the first two (Model 3); there was no further attenuation after further adjustments for exercise, smoking and alcohol consumption (Model 4) and diet (Model 5).

Of the 222 metabolites with no significant sex modification, 11 were associated with short-term verbal memory $(p<0.002)$ in sex-adjusted analyses (Fig. 2, Model $1)$. The strongest association was with the diameter of high-density lipoproteins (HDL-D) (beta $=0.156,95 \% \mathrm{CI}$ $\left.0.08-0.23, p=4.11 \times 10^{-5}\right)$. The rest of the associations were mainly with lipids in large and X-large HDLs, with the ratio of poly and mono-unsaturated FAs to total Fas (PUFA-FA and MUFA-FA, respectively), as well as with glycoprotein acetyl (GP). The effects of adjustments in the whole-sample analyses were similar to those observed in females, with most associations being attenuated in Model 3.

\section{Delayed verbal memory}

Two metabolite measures showed sex modification $(p<$ 0.1 ) in Model 1. These two measures, pyruvate and DHAFA, were associated with delayed verbal memory in females (Fig. 2, Model 1), the strongest association being with pyruvate (beta $=0.169,95 \%$ CI $0.07-0.27, p=1.00 \times$ $\left.10^{-3}\right)$. These associations remained statistically significant in Model 2 but were attenuated in Model 3, and there was no further attenuation in Models 4 and 5.

None of the rest of the 231 metabolite measures were associated with delayed verbal memory in sex-adjusted whole-sample analyses.

\section{Search speed}

Of the 40 metabolite measures with significant sex modification $(p<0.1)$ in Model 1 , eight were associated with search speed in men. The strongest association was with total cholesterol in intermediate density lipoprotein $\left(\right.$ IDL-C) $\left(\right.$ beta $=0.187,95 \%$ CI 0.08-0.29, $\left.p=4.21 \times 10^{-4}\right)$, and the rest of the associations were with the ratios of triglycerides (TG), cholesterol and cholesterol esters (ChoE) to intermediate and low density lipoproteins (IDLs and LDLs). These associations remained, albeit weakened, after adjustments $(p<0.05$, Model 5$)$.

In whole-sample sex-adjusted analyses, MUFA-FA and PUFA-FA were associated with search speed (Fig. 2; Model 1). The strongest association was with MUFA-FA $\left(\right.$ beta $=-0.113,95 \% \mathrm{CI}-0.18$ to $-0.04, p=0.1 .5 \times 10^{-3}$ ). These associations were attenuated in Model 2 and further weakened by additional adjustments.

Figure 3 shows the associations between all metabolites and cognitive outcomes at $p<0.05$; the cross-sectional associations between all metabolites and the cognitive outcomes are presented in Supplementary Table S4.

\section{Cognitive change between $60-64$ and 69 years}

Forty-three metabolites showed sex modification and one, the ratio of free cholesterol to total lipids in XL-HDL 


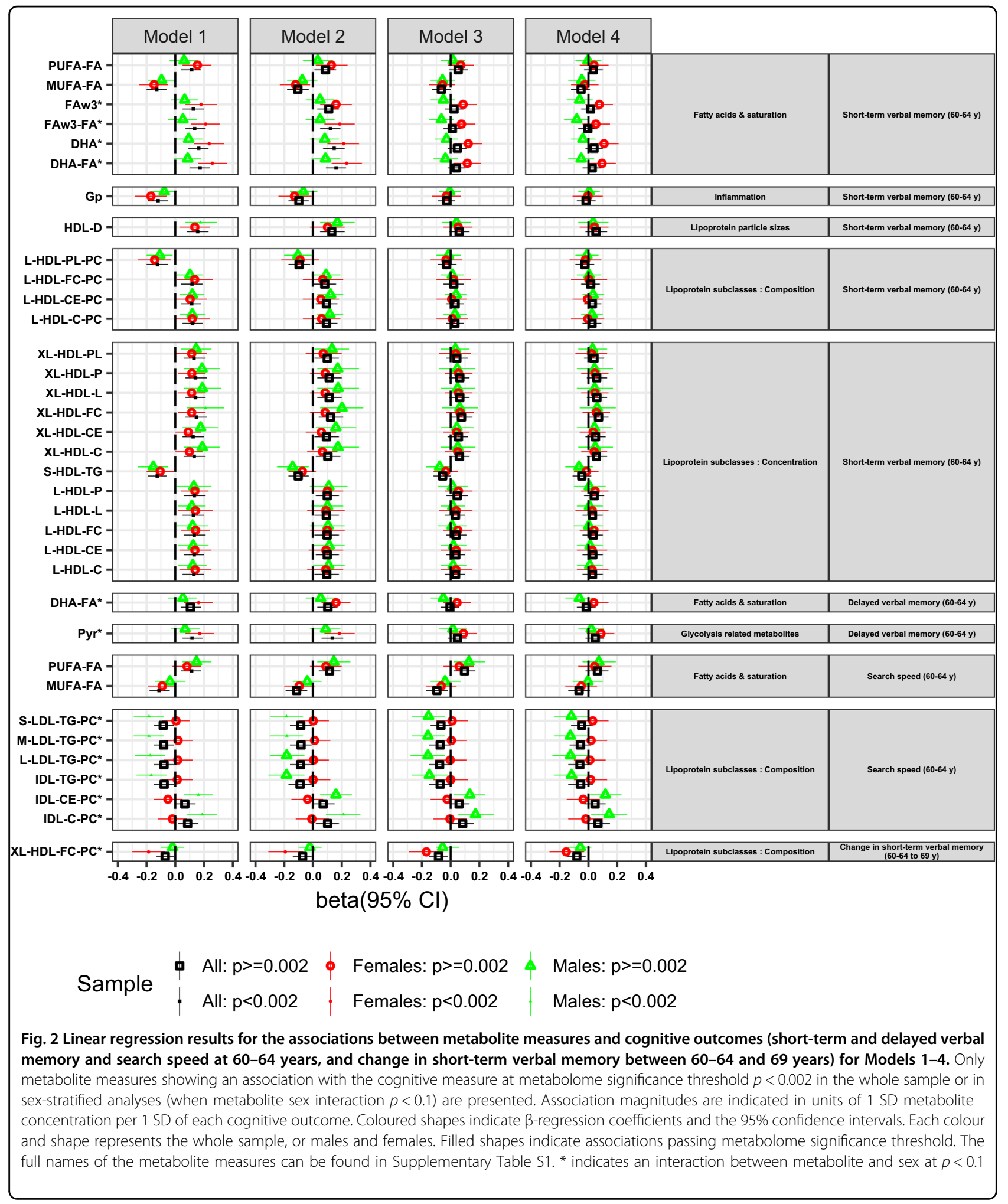

(XL-HDL-FC-PC), was associated with greater decline in short-term verbal memory in females (beta $=-0.186,95 \%$ CI -0.30 to $-0.07, p=1.31 \times 10^{-3}$ ), with slight attenuation in Model 3. No associations were observed between the rest of the 232 metabolites and change in short-term verbal memory in whole-sample sex-adjusted analyses.

Of the remaining 190 metabolites, 4 showed evidence of sex modification but none were associated with change in 


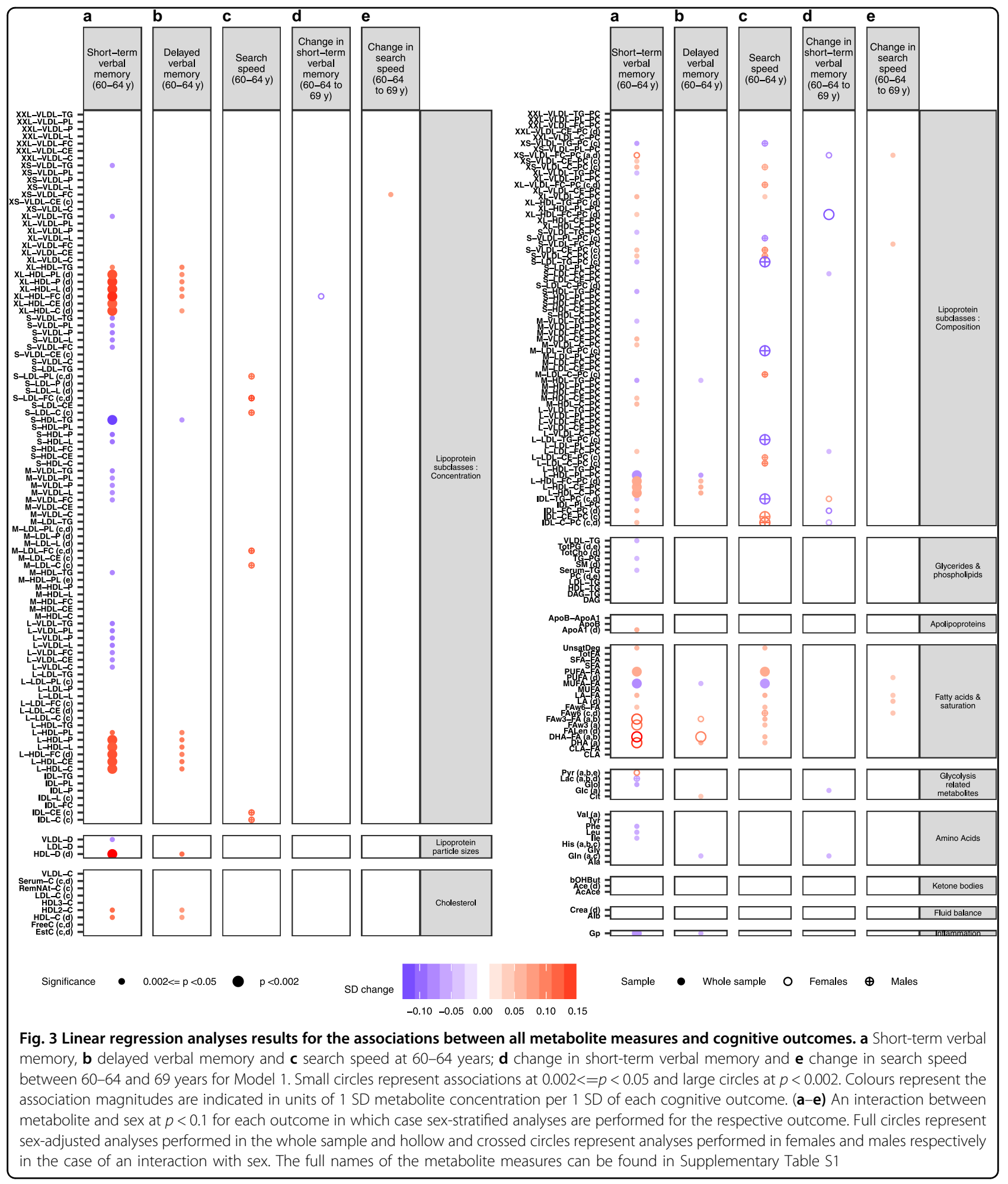

search speed between 60-64 and 69 years in sex-stratified analyses. There were no associations between the rest of the metabolites and change in search speed between 60-64 and 69 in whole-sample sex-adjusted analyses.
Figure 3 shows the associations between metabolites and change in cognition at $p<0.05$; the associations between all metabolites and change in cognition are presented in Supplementary Table S4. 


\section{Discussion}

Using a large British population-based birth cohort we investigated associations between 233 blood metabolites at 60-64 years, and memory and speed of processing at the same age, and change in these cognitive functions from 60-64 to 69 years. We observed associations between different metabolite classes, especially FA and lipoprotein sub-classes, and cognition, some of which were sex specific. These associations were independent of health and health-related behaviours but were largely explained by childhood cognition and education (Supplementary Table S2), particularly for PUFAs and HDLs.

This is to our knowledge the largest single study to systematically investigate how the midlife blood metabolome is associated with midlife cognition and cognitive decline, and how life course factors explain these associations. We used a representative British birth cohort study with metabolite data representing a broad molecular signature of systemic metabolism, and concurrent measures of memory and processing speed. Cognition was re-assessed at age 69, at a stage of the life course when pathophysiological changes relevant to CNS function are likely to be accumulating, but dementia is still rare. The main strength of our study is the range of potential confounders and mediators. These include rarelyavailable childhood cognitive ability, education, socioeconomic status, BMI, lipid medication, diet information, exercise and lifetime smoking and alcohol consumption information. Building sequential models we were able to interrogate the influence of all these covariables on the associations of metabolites with cognition. Our study also has some limitations. These include lack of metabolite data at age 69 , which precludes investigation of covariate changes in metabolite levels and cognition; and lack of childhood metabolite data, which disallows detailed investigation of life course bi-directionality between the metabolome and cognition. Further studies may investigate the latter using appropriate instrumental approaches such as Mendelian randomisation (MR).

With these strengths and limitations in mind, how should these findings be interpreted?

The adult human brain comprises about $20 \%$ of the whole body's cholesterol ${ }^{8}$ and contains the largest diversity of lipid classes. n3-PUFAs may optimise the efficiency and plasticity of synaptic transmission in the brain; may dampen glial-activated pro-inflammatory events caused by stress; and may promote the renewal of neuronal cells in the hippocampus ${ }^{21}$, a key structure for normal and abnormal cognitive ageing. Studies have shown associations between cognitive ageing and $\mathrm{AD}$ and DHA containing phosphatidylcholines (PCs) in blood ${ }^{3,22,23}$ and brain tissue ${ }^{24,25}$, as well as between DHA and general cognitive ability and dementia ${ }^{4}$, with Mfsd2a identified as the transporter of DHA through the blood-brain barrier $^{26}$. A number of studies have investigated the association of long chain PUFA (LC-PUFA) supplementation with $\mathrm{AD}$ and although results are overall inconsistent a recent meta-analysis of randomised control trials indicated that $\mathrm{n} 3$-PUFAs supplementation seems to have beneficial effects on systemic endothelial vasodilator function and cognitive function ${ }^{27}$. However, the concentration of essential FAs decreases in the ageing brain ${ }^{28}$. Mechanisms for this are uncertain, but likely include dietary changes, impaired desaturase activity, increased lipid peroxidation through impaired antioxidant systems, and impaired vascular health ${ }^{28}$. Here, we found positive associations with PUFAs and negative associations with MUFAs and cognition, with the associations of n3-PUFAs and DHA with cognition being observed only in women. This is consistent with previous work that has shown that the cognitive benefits of DHA were more profound in women $^{27}$. Although it has been reported that such sexspecific associations could be attributed to bmi differences between the two genders ${ }^{29}$, the association of DHA and cognition in women here was independent to bmi. Future studies will interrogate the complex interplay between $\mathrm{n} 3$ PUFA, cognition and gender.

Our study also points to associations between cognition and different lipid subfractions. These included associations between short-term verbal memory and subfractions of Large and XL HDLs, including ChoEs, PCs, TGs and free cholesterol, and associations between search speed and LDLs/IDLs subfractions, mainly TGs, in men. In addition to lipid transport, HDL regulates vascular health via mediating vasorelaxation, inflammation and oxidative stress, and promotes endothelial cell survival and integrity $^{30}$. Although studies are not always consistent, HDLs have been implicated in age related cognitive decline and $\mathrm{AD}$ (reviewed in ref. ${ }^{31}$ ) and were recently associated with general cognitive ability and dementia and $\mathrm{AD}^{4}$. Additionally, complex sex-specific associations between LDLs and IDLs and cognitive decline in older adults and $\mathrm{AD}$ have been previously reported ${ }^{32,33}$. However, Mendelian randomisation studies have shown no evidence of a causal association between HDL-C or LDL-C and $\mathrm{AD}^{34,35}$. Nevertheless, the association of these compositionally and functionally diverse lipoprotein particles in relation to cognition and potentially $\mathrm{AD}$ is not well understood and warrants further investigation. Interestingly, it has been suggested that PUFAs can alter serum lipid profile; ${ }^{36-39}$ serum omega- 3 and omega- 6 PUFAs have been associated with higher serum levels of large HDLs and HDL diameter and serum MUFA concentration has been inversely associated with Large HDL particles and positively associated with LDL particles. Indeed, here, we observed the same patterns of associations between serum lipid profiles and FAs (Supplementary Figure S1), although directionality was not established. 
Finally, we found negative associations between glycoprotein acetyls (mainly a1-acid glycoprotein) and shortterm verbal memory in the whole sample, and positive associations between pyruvate and delayed verbal memory in females. With regard to glycoprotein acetyls, changes in the level of several glycoproteins have been observed in the hippocampus and inferior parietal lobe in human $\mathrm{AD}^{40}$ some of these glycoproteins interact with neurofibrillary tangles, leading to speculation that changes in their glycosylation may be associated with the pathogenesis of this disease ${ }^{40}$. Additionally, A1-acid glycoprotein was previously found to be a strong predictor of 10 -year mortality ${ }^{41}$ and was also recently negatively associated with general cognitive ability ${ }^{4}$. Little is known about human cognition in relation to to pyruvate levels, but the present findings are consistent with relevant animal studies. For example, rodent models of $\mathrm{AD}$ suggest apparent neuroprotective effects of pyruvate administration; mechanisms include protection against beta amyloid oligomer-induced neuronal cell death ${ }^{42}$, and (consistent with essential FAs) reduction of lipid peroxidation and oxidative stress ${ }^{43}$.

An important and consistent finding from this study is that associations between metabolites, in particular FAs, and cognitive function, in particular verbal memory, were largely explained by childhood cognition and educational attainment. Indeed, we found one example of a stronger association for childhood cognition than adult cognition (n3-PUFAs in men (Supplementary Table 2)). With regard to childhood cognition, this is positively associated with healthy dietary choice in NSHD, even after taking account of education and lifetime $\mathrm{SEP}^{44}$. However, health behaviours were not important explanatory variables in this study (Supplementary Tables S3a-c) even though they were associated with FAs (Supplementary Table S2). Alternatively, this association may also reflect lifetime bidirectionality between FAs and cognition, beginning with maternal FA intake, which prolongs the duration of pregnancy $^{45}$ (leading to heavier birthweight, itself positively associated with cognitive development ${ }^{46}$ ). FAs then cross the placenta, and separately enter breast milk. Breastfeeding, in turn, is positively associated with cognitive development, even when confounding from maternal cognitive ability is controlled ${ }^{47}$. From this perspective, adjusting for childhood cognition could be regarded as over-adjustment rather than removing a source of confounding; however, incorporating the interplay between early growth, nutrition and cognitive development, and how these influence later metabolite status and cognitive function, is beyond the scope of this study.

A third possibility is that the link between childhood cognition and metabolites is underpinned by a common genetic cause or by a combination of genetic and dietary or sex influences. Variation in FA desaturase (FADS) 1/2 genes, which influence rate of n3-PUFAs and n6-PUFA synthesis, contributes to blood concentrations of FAs ${ }^{48,49}$, with heritability estimates accounting for $32-70 \%$ of FA variation $^{48,50,51}$. Complex interactions between FADS genotypes and maternal and infant dietary intakes and LC-PUFAs concentrations have been described, with maternal genetic variation in FADS frequently associated with lower concentrations of LC-PUFAs in maternal and infant blood and in breast milk ${ }^{52-54}$. Additionally, the breastfeeding effects on childhood cognition have been reported to be modified by FADs genes ${ }^{55-58}$, although studies are not always consistent ${ }^{59,60}$. Finally, a recent MR study has reported the effects of FADS genotypes on cognition in 8-11 years old schoolchildren to be sex specific $^{61}$.

\section{Conclusion}

Findings from this study improve our understanding of the peripheral metabolic processes underlying cognitive ageing. Our study suggests that the levels of circulating metabolites in midlife, in particular FAs and different lipid sub-classes, are associated with midlife cognition, and that some of these associations are sex specific. The attenuation of these associations, after taking into consideration childhood cognition and education, suggests that the metabolic profile may be altered earlier in the life course, conferring lifetime vulnerability to poor cognition. This highlights how adding life course information helps our understanding of these associations, which could have been otherwise overestimated in midlife. As metabolites are potentially modifiable markers through diet and lifestyle, these findings could hold special value in cognitive ageing research, and may contribute to risk-reduction strategies for cognitive impairment and dementia.

\footnotetext{
Acknowledgements

PP is a UCL Springboard Population Science Fellow. We would like to acknowledge Vincent Plagnol for funding the assays, Jonathan Griffiths for the help with the NMR data management and Aroon Hingorani for coordinating the University College London-London School of Hygiene and Tropical Medicine-Edinburgh-Bristol (UCLEB) Consortium metabolomics. We thank NSHD study members who took part in this and previous data collections, for their continuing support. We thank staff at the following centers: MRC Unit for LHA, MRC Human Nutrition Research, Cambridge; MRC Epidemiology Unit, Cambridge; MRC Epidemiology Resource Centre, Southampton; Welcome Trust (WT) Clinical Research Facility (CRF) Manchester, the Manchester Heart Centre and the Department of Clinical Radiology at the Central Manchester University Hospitals NHS Foundation Trust; WTCRF, Medical Physics and the Department of Cardiology at the Western General Hospital in Edinburgh; WTCRF, Department of Nuclear Medicine and the Department of Cardiology at University Hospital Birmingham; WTCRF and the Department of Nuclear Medicine at University College London Hospital; CRF, the Department of Medical Physics and the Department of Cardiology at the University Hospital of Wales; CRF and Twin Research Unit at St Thomas' Hospital London. We thank the team at Kantar Public (formerly known as TNS BMRB) and Medicals Direct Group (MDG) for their work in project development, management of the nurse field-force and data collection and delivery. This work was supported by the UK Medical Research Council [MC_UU_12019/1, MC_UU_12019/2,
} 
MC_UU_12019/3] which provides core funding for the MRC National Survey of Health and Development.

\section{Conflict of interest}

The authors declare that they have no conflict of interest.

\section{Publisher's note}

Springer Nature remains neutral with regard to jurisdictional claims in published maps and institutional affiliations.

Supplementary Information accompanies this paper at (https://doi.org/ 10.1038/s41398-018-0253-0).

Received: 13 November 2017 Revised: 25 April 2018 Accepted: 11 May 2018

Published online: 26 September 2018

\section{References}

1. (2008). FMCaWP, summary. FPrE, The Government Office for Science L.

2. Beger, R. D. et al. Metabolomics enables precision medicine: "A White Paper, Community Perspective". Metabolomics 12, 149 (2016).

3. Simpson, B. N. et al. Blood metabolite markers of cognitive performance and brain function in aging. J. Cereb. Blood Flow. Metab. 36, 1212-1223 (2016).

4. van der Lee, S. J. et al. Circulating metabolites and general cognitive ability and dementia: evidence from 11 cohort studies. Alzheimers Dement. 14, 707-722 (2018)

5. Richards, M. \& Sacker, A. Lifetime antecedents of cognitive reserve. J. Clin. Exp. Neuropsychol. 25, 614-624 (2003).

6. Kuh, D. et al. The MRC National Survey of Health and Development reaches age 70: maintaining participation at older ages in a birth cohort study. Eur. J. Epidemiol. 31, 1135-1147 (2016).

7. Stafford, M. et al. Using a birth cohort to study ageing: representativeness and response rates in the National Survey of Health and Development. Eur. J. Ageing 10, 145-157 (2013).

8. Shah, T. et al. Population genomics of cardiometabolic traits: design of the University College London-London School of Hygiene and Tropical MedicineEdinburgh-Bristol (UCLEB) Consortium. PLoS ONE 8, e71345 (2013).

9. Soininen, P., Kangas, A. J., Wurtz, P., Suna, T. \& Ala-Korpela, M. Quantitative serum nuclear magnetic resonance metabolomics in cardiovascular epidemiology and genetics. Circ. Cardiovasc. Genet. 8, 192-206 (2015).

10. Soininen, P. et al. High-throughput serum NMR metabonomics for costeffective holistic studies on systemic metabolism. Analyst 134, 1781-1785 (2009).

11. Richards, M. et al. Lifetime affect and midlife cognitive function: prospective birth cohort study. Br. J. Psychiatry 204, 194-199 (2014).

12. Wurtz, P. et al. Metabolic signatures of adiposity in young adults: Mendelian randomization analysis and effects of weight change. PLoS Med. 11, e1001765 (2014).

13. Holmes, E. et al. Human metabolic phenotype diversity and its association with diet and blood pressure. Nature 453, 396-400 (2008).

14. Krug, $\mathrm{S}$. et al. The dynamic range of the human metabolome revealed by challenges. FASEB J. 26, 2607-2619 (2012).

15. Stella, C. et al. Susceptibility of human metabolic phenotypes to dietary modulation. J. Proteome Res. 5, 2780-2788 (2006).

16. Wurtz, P. et al. Metabolic profiling of alcohol consumption in 9778 young adults. Int. J. Epidemiol. 45, 1493-1506 (2016).

17. Pidgeon, DA. in The home and the school. (ed Douglas, J.W.B.) (Macgibbon \& Kee, London, 1964).

18. Nishida, A. et al. Adolescent self-organization and adult smoking and drinking over fifty years of follow-up: The British 1946 Birth Cohort. PLOS ONE 11, e0146731 (2016).

19. Pastorino, S., Richards, M., Pierce, M. \& Ambrosini, G. L. A high-fat, highglycaemic index, low-fibre dietary pattern is prospectively associated with type 2 diabetes in a British birth cohort. Br. J. Nutr. 115, 1632-1642 (2016).

20. Sterne, J. A. et al. Multiple imputation for missing data in epidemiological and clinical research: potential and pitfalls. BMJ 338, b2393 (2009).
21. Denis, I., Potier, B., Vancassel, S., Heberden, C. \& Lavialle, M. Omega-3 fatty acids and brain resistance to ageing and stress: body of evidence and possible mechanisms. Ageing Res. Rev. 12, 579-594 (2013).

22. Whiley, L. et al. Evidence of altered phosphatidylcholine metabolism in Alzheimer's disease. Neurobiol. Aging 35, 271-278 (2014).

23. Schaefer, E. J. et al. Plasma phosphatidylcholine docosahexaenoic acid content and risk of dementia and Alzheimer disease: the Framingham Heart Study. Arch. Neurol. 63, 1545-1550 (2006).

24. Varma, V. R. et al. Brain and blood metabolite signatures of pathology and progression in Alzheimer disease: a targeted metabolomics study. PLoS Med. 15, e1002482 (2018).

25. Snowden, S. G. et al. Association between fatty acid metabolism in the brain and Alzheimer disease neuropathology and cognitive performance: a nontargeted metabolomic study. PLoS Med. 14, e1002266 (2017).

26. Nguyen, L. N. et al. Mfsd2a is a transporter for the essential omega-3 fatty acid docosahexaenoic acid. Nature 509, 503-506 (2014).

27. Kuszewski, J. C., Wong, R. H. X. \& Howe, P. R. C. Effects of long-chain omega-3 polyunsaturated fatty acids on endothelial vasodilator function and cognitionare they interrelated? Nutrients $\mathbf{9}, 5$ (2017).

28. Uauy, R. \& Dangour, A. D. Nutrition in brain development and aging: role of essential fatty acids. Nutr. Rev. 64, S24-S33 (2006). discussion S72-91.

29. Lohner, S., Fekete, K, Marosvolgyi, T. \& Decsi, T. Gender differences in the longchain polyunsaturated fatty acid status: systematic review of 51 publications. Ann. Nutr. Metab. 62, 98-112 (2013).

30. Stukas, S., Robert, J. \& Wellington, C. L. High-density lipoproteins and cerebrovascular integrity in Alzheimer's disease. Cell. Metab. 19, 574-591 (2014).

31. Hottman, D. A., Chernick, D., Cheng, S., Wang, Z. \& Li, L. HDL and cognition in neurodegenerative disorders. Neurobiol. Dis. 72, 22-36 (2014).

32. Ancelin, M. L. et al. Gender-specific associations between lipids and cognitive decline in the elderly. Eur. Neuropsychopharmacol. 24, 1056-1066 (2014).

33. Ancelin, M. L. et al. Sex differences in the associations between lipid levels and incident dementia. J. Alzheimers Dis. 34, 519-528 (2013).

34. Ostergaard, S. D. et al. Associations between potentially modifiable risk factors and Alzheimer disease: a Mendelian randomization study. PLoS Med. 12, e1001841 (2015).

35. Proitsi, P. et al. Genetic predisposition to increased blood cholesterol and triglyceride lipid levels and risk of Alzheimer disease: a Mendelian randomization analysis. PLoS Med. 11, e1001713 (2014).

36. Balk, E. M. et al. Effects of omega-3 fatty acids on serum markers of cardiovascular disease risk: a systematic review. Atherosclerosis 189, 19-30 (2006).

37. Ferrucci, L. et al. Relationship of plasma polyunsaturated fatty acids to circulating inflammatory markers. J. Clin. Endocrinol. Metab. 91, 439-446 (2006).

38. Jelenkovic, A. et al. Association between serum fatty acids and lipoprotein subclass profile in healthy young adults: exploring common genetic and environmental factors. Atherosclerosis 233, 394-402 (2014).

39. Motoyama, K. R. et al. Association of serum n-6 and n-3 polyunsaturated fatty acids with lipids in 3 populations of middle-aged men. Am. J. Clin. Nutr. 90, 49-55 (2009).

40. Butterfield, D. A. \& Owen, J. B. Lectin-affinity chromatography brain glycoproteomics and Alzheimer disease: insights into protein alterations consistent with the pathology and progression of this dementing disorder. Proteom. Clin. Appl. 5, 50-56 (2011).

41. Fischer, K. et al. Biomarker profiling by nuclear magnetic resonance spectroscopy for the prediction of all-cause mortality: an observational study of 17,345 persons. PLoS Med. 11, e1001606 (2014).

42. Wang, X., Hu, X., Yang, Y., Takata, T. \& Sakurai, T. Systemic pyruvate administration markedly reduces neuronal death and cognitive impairment in a rat model of Alzheimer's disease. Exp. Neurol. 271, 145-154 (2015).

43. Isopi, E. et al. Pyruvate prevents the development of age-dependent cognitive deficits in a mouse model of Alzheimer's disease without reducing amyloid and tau pathology. Neurobiol. Dis. 81, 214-224 (2015).

44. Richards, M., Stephen, A. \& Mishra, G. Health returns to cognitive capital in the British 1946 birth cohort. Longitud. Life Course Stud. 1, 281-296 (2010).

45. Decsi, T. Effects of supplementing LCPUFA to the diet of pregnant women: data from RCT. Adv. Exp. Med. Biol. 646, 65-69 (2009).

46. Shenkin, S. D., Starr, J. M. \& Deary, I. J. Birth weight and cognitive ability in childhood: a systematic review. Psychol. Bull. 130, 989-1013 (2004).

47. Horta, B. L., Loret de Mola, C. \& Victora, C. G. Breastfeeding and intelligence: a systematic review and meta-analysis. Acta Paediatr. 104, 14-19 (2015). 
48. Kettunen, J. et al. Genome-wide association study identifies multiple loci influencing human serum metabolite levels. Nat. Genet. 44, 269-276 (2012).

49. Tanaka, T. et al. Genome-wide association study of plasma polyunsaturated fatty acids in the InCHIANTI Study. PLoS Genet. 5, e1000338 (2009).

50. Harris, W. S. et al. Clinical correlates and heritability of erythrocyte eicosapentaenoic and docosahexaenoic acid content in the Framingham Heart Study. Atherosclerosis 225, 425-431 (2012).

51. Lemaitre, R. N., Siscovick, D. S., Berry, E. M., Kark, J. D. \& Friedlander, Y. Familial aggregation of red blood cell membrane fatty acid composition: the Kibbutzim Family Study. Metabolism 57, 662-668 (2008).

52. Koletzko, B., Lattka, E., Zeilinger, S., Illig, T. \& Steer, C. Genetic variants of the fatty acid desaturase gene cluster predict amounts of red blood cell docosahexaenoic and other polyunsaturated fatty acids in pregnant women: findings from the Avon Longitudinal Study of Parents and Children. Am. J. Clin. Nutr. 93, 211-219 (2011).

53. Lattka, E. et al. Umbilical cord PUFA are determined by maternal and child fatty acid desaturase (FADS) genetic variants in the Avon Longitudinal Study of Parents and Children (ALSPAC). Br. J. Nutr. 109, 1196-1210 (2013).

54. Molto-Puigmarti, C. et al. FADS1 FADS2 gene variants modify the association between fish intake and the docosahexaenoic acid proportions in human milk. Am. J. Clin. Nutr. 91, 1368-1376 (2010).
55. Caspi, A. et al. Moderation of breastfeeding effects on the $1 \mathrm{Q}$ by genetic variation in fatty acid metabolism. Proc. Natl Acad. Sci. USA 104, 18860-18865 (2007).

56. Morales, E. et al. Genetic variants of the FADS gene cluster and ELOVL gene family, colostrums LC-PUFA levels, breastfeeding, and child cognition. PLoS ONE 6, e17181 (2011)

57. Steer, C. D., Davey Smith, G., Emmett, P. M., Hibbeln, J. R. \& Golding, J. FADS2 polymorphisms modify the effect of breastfeeding on child IQ. PLOS ONE 5, e11570 (2010).

58. Steer, C. D., Hibbeln, J. R., Golding, J. \& Davey Smith, G. Polyunsaturated fatty acid levels in blood during pregnancy, at birth and at 7 years: their associations with two common FADS2 polymorphisms. Hum. Mol. Genet. 21, 1504-1512 (2012).

59. Groen-Blokhuis, M. M. et al. A prospective study of the effects of breastfeeding and FADS2 polymorphisms on cognition and hyperactivity/attention problems. Am. J. Med. Genet. B. Neuropsychiatr. Genet. 162B, 457-465 (2013).

60. Martin, N. W. et al. Cognitive function in adolescence: testing for interactions between breast-feeding and FADS2 polymorphisms. J. Am. Acad. Child Adolesc. Psychiatry 50, 55-62 (2011).

61. Lauritzen, L. et al. Mendelian randomization shows sex-specific associations between long-chain PUFA-related genotypes and cognitive performance in Danish schoolchildren. Am. J. Clin. Nutr. 106, 88-95 (2017). 